\title{
Namenregister
}

Abbott 5076.

Abelin 5062.

Abrams 5027.

Adams 5115.

Afanassjewa 5046 .

Ahlén 5123.

Aikasjan 4978.

Aka 4982.

Aktiebolaget Leo 5082.

Aktiebolaget

Pharmacia 5078.

Alberto 5070.

Albrecht 4974 .

Allard 4974.

Allegheny Ludlum

Steel Corp. 5102

Allen, I. G. 5073.

Allen, P. W. 5043.

Allied Chemical

\& Dye Corn.

5107.

Alves 5091.

American Chemica

Paint Co. 5104.

American Cyanan Co. 5081. 5112 . 5115.

American Viscose Corp. 5124.

Anderson jr., A. G. 5021.

Anderson, V.D., Co. 5121 .

Andrejew 5010 .

Andreoni 5062

Anglo-Iranian $\mathrm{O}$

Co. Ltd. 5113.

Antipenko 5073.

Antonow 4988 .

Appel 5076 .

Arbusow 4995.

Arcus 5017.

Armbruster 5068

Armour \& Co. 5

Armour Research

Foundation 50

Armstrong 5030.

Arnaldi 5033 .

Arneson 5068.

Arnoldsson 5061.

Arnstein 5008.

Artom 5119.

Arutjunjan 5073 .

Arvy 5064 .

Arzac 5089.

Asarch 5053.

Asaro 4970 .

Ashley 5076.

Aspden 4976.

Asplund 5123.

Assenmacher 5060 .

Aubertin 5074

Ausilio, d' 5098 .

Austerweil 5043.

Badische Anilin.

\& Soda-Fabrik

5077. 5091. 5092 . 5094.

5113.

Bailey 5034 .

Bakelite Corp. 5114 .

Bakelite

G. m. b. H. 5112 .

Ballabio 5063.

Ballay 5100 .

Balmain 5063.

Bansi 5061.

Baranowskaja

Barber 4989 .

Barett 5068.

Barker 5088 .

Barnett 4975 .

Barocas 4971

Barr, de 4976.

Barraud 5060 .

Barré 5017.

Barsch 4974.
Barthelheimer 5071. British Oxygen Co. Cirilli 4989. Bartenew 5044. Ltd. 5105.

Barthel, Michael, Brockmann, H. \& Co., Chemische 7992 . und Mineralöl- Brockmann, $\mathbf{R}$. Werke, G. m. b. H. 4985 5111.

Battaglini 5118 .

Broda 4977

Bauer 5068 .

Baur 5112.

Baxter 5083.

Beck 5106.

Brodhun 5041.

Brodie 4969.

Brooksby 5057.

Broustet 5065 .

Beck, Koller \& Co. Bruckshaw 4990.

Ltd. 5112.

Beckey 4979.

Begemann 5063.

Belenki 4966.

Bellavita 5023.

Bentley 5008 .

Berg 5096.

Bergen, Van 5073.

Berger, I R. 5068.

Berger, Lewis,

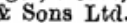

5114.

Bruckshaw 4990.

Bucha 5059.

Buchanan 4999.

Buchegger 5063.

Buchert 5047.

Buck 5085 .

Buckler 5115.

Buckley 5073 .

Budde 4992.

Budnick 5052 .

Budnikow 5096.

Buerger 4982.

Berger, R. E. 5064. Burdese 4989

Berger, S. 5051.

Bergkvist 5000.

Bergmann 4966.

Bergwerksverband zur Verwertung von Schutzrechten der Kohlentechnik G.m. b. H. 5099.

Beringer 4972 .

Berlincourt 4975 .

Berlowitsch 4969 .

Bernard 5065 .

Bernhardt 4989 .

Bertaut 4983.

Beythien 5048.

Bieleck 4983.

Biesele 5051.

Binet 5065 .

Binon 5024 .

Birkel 5098 .

Birkinshaw 5037.

Blacizek 5066 .

Black 5071 .

Blix 5060 .

Bllavita 5023 .

Blau 5108.

Boberg 5077 .

Bode 5485 .

Boeger 5067.

Böhler, Gebr. \& Co. Akt.-Ges. 5102.

Boiko 5097.

Boki 4997.

Boldebuck 4986 .

Boltz 5085 .

Bombelli 4988.

Bonafos 5072 .

Bonebrake 5068 .

Borchert 5044.

Borkowski 5011.

Boss 5097.

Botden 5092.

Bothner-By 5000 .

Bottini 4118.

Boucher 5091.

Bowen 5092.

Bozorth 4976.

Bracken 5037.

Bradish 5057.

Burley 5077.

Burriel 5086.

Busby 5120 .

Bush 5122.

Buskirk, van 5066 .

Butler, J. A. W. [5052].

Butzeroga 5097.

Buu-Hoī 5024. 5055

Buy, Du 5049 .

Califano 5057 .

Calligari 5064.

Callow 5032.

Campbell 5088.

Campo de Hachen, del 5062 .

Canadian General Electric Co. Ltd. 5107.

Canavan 4969.

Cantù 5073.

Capitani 4999, 5085 .

Carboni 5033.

Carlson, S. 5055 .

Carpenter 5008.

Carr 5070.

Carrancio 4977.

Carrante 5097.

Carroll 4990.

Casida 5071.

Cassella Farbwerke

Mainkur 5080 .

Cassen 5068.

Cauer 5078.

Caule 5083.

Cavalli, C. A. [4980].

Cavalli, L. L. 5056 Celanese Corp. of America 5113.

Cennamo 4998.

Chapman 5012.

Chatfleld 5110 .

Cheatham 5043.

Chemical Works of Richter Gedeon 5079 .

Chemische Werke Albert 5099.

Cheng 4969.

Cheymol 5077.

Brandt [4965].

Bratring 5123 .

Brauer 4985 .

Breit 4968.

Brenner 5089.

Breshnew 5097.

Brewerton 5069.

Brian 5055 .

Brice 5075 .

Cities Service

Clark 5027

Clarke 5051 .

Clayson 4974.

ohn 4971.

Cole 4982 .

Collen 5100.

Combee 5092.

5104.

Compton 4993

Comsa 5061.

Comuzzi 5117.

Conradi 4995.

Corboud 5072 .

Cork 4967.

Cornforth 5032.

Cottrell 5101.

Cox, Ch. R.

5093 .

Cozzi 4978.

Cramer 5044.

Cristol 5019.

Daley 5061

Dauben 5031.

Dies 4981 .

Dean 4972

Dechary 5018.

Collins jr. 5093.

giques Evans jr. 4983.

camarge 5103 .

ox, R. E. 5013.

Dabagian 4970 .

Dammann 4973.

Darquennes 5097

Delapis 5018.

Desmoras 5097.

Destefanis 5070 .

Deutsche Gold-

\& Silber-Scheide-

anstalt vormals

Deutsche Hoff- 
Henkel \& Cic. G. m. b. H.

Henne 4999 .

Hennig 5069 .

Henry 4977.

Henseler 5065 .

Hérold 4988.

Herrmann 4968.

Hersant 5075 .

Hess 5122 .

High Duty Alloys Ltd. 5103 .

Hillebrand [5090].

Hittmalr 4967 .

Hochhauser 5066 .

Höppner 5095 .

Hoesch Akt.-Ges. 5112.

Hofer 4998.

Hofmann 5055 .

Holbrook 5023.

Holliday 4981.

Hollström 5100 .

Hollyer jr. 4973.

Holmes 5063.

Honig 4972.

Hopkins, E. G. 5092.

Hopkins, R. H. 5117 .

Horn 5077 .

Houdremont 5100 .

Houle 5066 .

Houtman 5092 .

Howard 5056 .

Howland 4979 .

Howlett 5000 .

Hoyer 4994.

Hoyt 5124.

Hudgens jr. 4970 .

Hüsselmann 5061 .

Hüttenwerk Hörde

Akt.-Ges. 5099.

Hüttenwerk

Huckingen

Akt.-Ges. 5099.

Huff 5054

Hughes, C. R. 5068 .

Hughes, I. W. 5088 .

Hull jr. 4968 .

Humenik jr. 5096.

Hunting 4973.

Hurtado Acera

Hutchins 5122 .

Hutchinson j.

Hyde 5085 .

Imai 4990.

Imperial Chemical Industrles Ltd. 5105.

Ingold, C. K. 4971 . 4997.

Interchemical Corp. 5111.

International

Junkins 4989.

Jura 4979

Juutinen 5087.

Juvonen 5122 .

Kahan 5016.

Kaiser 5075 .

Kalb 4984.

Kalvoda 5076

Kane 5070 .

Kanngiesser 5058.

Kardon 4996.

Karlovac 5075 .

Kasanski 5002.

Katajewa 4995

Kaufman 5042.

Kaufmann 5110 .

Kenty 5083.

Kenyon 5000 .

Khan 5001.

Kidder 5071.

Kiefer 5085 .

Kiese 5053 .

Kilchling 5063.

Kilp 5116.

Kimbel 5034.

King, G. W. 4997.

King, S. J. 5074 .

Kirk 5031.

Kirrmann 4995.

Kivelson 4971

K! lein 5056.

Kliemann 5070 .

Klykow 5048.

Knibbs 5096 .

Knight 5057 .

Koch 5066 .

Kock 5119.

Köhler 5054.

Koenen 5118 .

Koepsell 5087 .

Kolthoff 5084 .

Komissarowa 4988

Kononow 5013.

Koppers Co., Inc. 5108.

Koppers, Heinrich, Lyons 5064.

G. m. b. H. 5094. McBee 5004 .

Korhonen 4984.

Koritz 5089.

Kornberg 5053.

Kosak 5023.

Kotanl 4971.

Kotschetkow 5007.

Koutz 5070.

Kraus 4977.

Kressman, T. R. E. 4982. 5090 .

Krewson 5075 .

Kritchevsky 5031

Kröger 5095.

Kudrjawzew 4989 .

Kurbatov 4969.

Kurssanow 5015

Kurtew 5018.

Plant Kurylenko 4990. Co. Ltd. $5092 . \quad$ Kusminski 5045.

International Nickel Laboratoires Co., Inc. 5103.

Irvine 5100 .

Iwanenko 4967.

Iwanow, B. W. 5095 .

Francais de

Chimiothérapie

$$
5082 .
$$

Laffitte 4994.

Lambertson 5096 .

Iwanow, N. P. 5086. Lampe 5082.

Jackson, M. 4980. Landsberg 4975.

Jackson, M. D. 5101. Lane 5011.

Jahn 5064 .

Lange 4965 .

Lanza 5097.

Jensen, C. C. 5062. Laporte 4973.

Jensen, E. A. 5082. Lappin 5013.

Jensen, P. 4968 . Lapschina 5013.

Jerome 4974.

Jesserer 5066 .

Joffe 4966.

Johann 5085 .

Latham jr. 5015 .

Jawrence 5054 .

Lawrow 4989.

Leach 5016 .

Leandri 4994.

Johnson, J. S. 4977. Lebedew 4967.

Johnson, R. A. 5084. Le Bihan 5024.

Johnson, W. S. 5082. Lechat 5077

Jones, J. G. 5058 . Lechevallier 5055.

Jones, R. G. 5025 .

Jonker 5092.

Jordan 5042 .

Josien 4994

Jünger 5118 .

Lederer 5083.

Lee 4972.

Leishman 5072 .

Lendle 5074 .

Lenti 5052 .

Linke 5069 .
Le Quesne 5009.

5010 .

Lettré 5050 .

Leshnew 5045.

Lebire 5097 .

ver Brothers

\& Unilever Ltd. 5121.

Levi 4970.

Li, Ch. H. 5061.

Li, N. C. 4995.

Liber 5101.

Liebert 5054 .

Lincke 5100.

Lindroth 5095 .

Lipatow 5045 .

Lippold 5088 .

Lisunow 4990 .

Littlejolnn 5067.

Livingstone 5073 .

bo Aza 5090 .

Logan 5122.

Lohmeyer 5061 .

Lominski 5057 .

Lommer 5077 .

onza Elektrizitätswerke und Che mische Fabriken Akt.-Ges. 5099.

Loshaek 5043.

Loveless 5070 .

Lovera 4966.

Lu ie 5075 .

Lucien 5020 .

Luckmann 5120 .

Lundell [5090.]

Lunenfeld 5061 .

Lurie 5074.

unsis 5098 .

Lutter 5078 .

McClure 5068.

MacDonald, D. M. 5030.

McDonald, L. E. 5071.

McEwen 5001

McGeachin 5013.

MacGillivray 5027.

McGllvery 5028.

Macher 5116.

McIntosh 5063.

Mack 5102.

Mackenzie 5116.

McSweeney 5006.

Maleyran 5024.

Malitson 4973.

Mandeville 4974.

Manufactures de

Produits Chimt ques du Nord Etablissements

Kuhlmann 5099.

Maravall Casesnove 4970.

Marbet 5065.

Marcuse 5120.

Marconi 5087.

Marinari 5098.

Markon 4985

Marti 5063.

Martinet, A. 4973.

Martinet, J. 4973.

Martinez 4983.

Marvin 5042.

Marzluff 5004.

Mason 4992.

Mathe 5065 .

Mathieu, F. 5103.

Mathleu, H. [5090]

Matschinski 4983.

Mattill 5066.

Matzel 5069.

Maximow 4966.

May 5014. 5015.

Maymone 5118 .

Medalia 4987.

Meek 5019.

Mehta 5001.

Meier, C. 5111.

Meler, R. 5063.

Meinl 5049.

Mejersson 5045.

Melchlor 5069.

Melis 5098.

Melnick 5120.

Merck, E., Chemische

Fabrlk 5117.

Merz 5065.

Mesley 5017

Metro-Cutanit Ltd. 5104.

Meyer, W. 5073.

Michael 5037.

Michel-Lévy 4990 .

Midgley 4978 .

Miles 5055 .

Miller 4971.

Milton 4999 .

Minatschew 5002 .

Mingrino 5062 .

Mönnlg 5082 .

Mohlman 5093.

Iohr $\mathbf{5 1 1 8 .}$

Monsanto Chemical Co. 5113. 5114.

TontecatiniSoc. Gen.

Mineraria 
Slemens-Plania Akt.-Ges. für Kohlefabrikate Werk Meltingen 5113.

Ssllin 4967.

Ssinjakowa 5086 .

Ssologubowa 5096.

Standard Oil

m. b. H. 5092 .

Sllberman, R. 5074.

Silver 5044 .

Stanolind Oll \& Gas Thamsen 5052 .

Silverman, L. 5090, Co. 5105.

Simchen 4987.

Simon, A. 5122 .

Slmon, J. 5071.

Singal 5067.

Skaller 5060

Stearns 5122

Steinhaus 5072

Stelnorth 5071 .

Stepanenko 5046 .

Stephan 5118

Skinner, H. A. 4996. Stephens 4973.

Sklnner, W. A. 5034. Stephenson 4993.

Skoog 5089. Sterligow 5002.

Slayter 5095

Smart [5048].

Stevens 5017.

Steward 5083 .

Stickings 5035 .

Smidth, F. L., \& Co. Stitch 4972.

A/S. 5096. Straumanis 4982.

Smlrnowa 5060

Smlth, D. D. 5057. Sumner [5054].

Smith, D. F. 4972. Sundstrom 5122.

Smith jr., E. P. 5071. Superior Sugar

Smith, J. A. 5064. Reflning Co. 5120.
Smith, N. L. 5028. Sutherland 4993.

Smith, N. L. 5028. Sutherland 4993.
Sneddon 5072.

Snyder 5124.

Svenner

Soc. An. d'Innova- Swallow 5001. tions Chimiques Swartz 4973.

Sinnova ou Sadlc Swift 5085 . 5106.

Solis 5062 .

Solomon 5065 .

Somers [5054].

Sousa, de 5086 .

Swift \& Co. 5121.

Sydenstricker

5067.

Sydow 5119 .

Szware 5114.

Spek, van der 4992. Tanskanen 5087 .

Spensley 5032 .

Spicer 5054 .

Spindler 4973.

Spoon 5075.

Spruch 4968.

Ssedletzki 4991.

Ssementschenko 4978.

Theden

Thiele 5075 .

Thyssen-Hütte, 5099.

Tibaldi 5057 .

Tichomirow 5073 .

Tietze 5067

Ting 5073.

Tkatsch 5071

Todd 5059 .

Tönjes 5122.

Tombs 4982.

Tomula 5087 .

Tonuttt 5062.

Torstl 5119.

Toulson 5071.

Tozzola 5057 .

Trischka 4973.

Troponwerke 5079 .

Tschenzowa 4974.

Tschufarow 4988.

Tullis [5068].

Turner 4973.

Uberall 4967.

Ulberg 5071 .

Ulrich 5011. 5099.

Tarbell 5016. Tatljewskaja 4988.

Taurins 5020.

Tawadse 5058 .

Teltelbaum 4980 .
5094. 5106. 5124. Terrill 5110.

Telefunken Gesell- United States of Wacker, Dr. AlexAmerica Secretary ander, Ges.für of Agriculture 5114. United States elektrochemische Industrle G.m.b.H. 5123 Rubber Co. 5108. Wagner, E. C. 5011. White, W. F. 5114. 5115. 5116. Wagner, K. W.

Universal Oil [4986]

[4986]. Whltman 5101 .

4987. Wagner-Jauregg

Thomas, R. 5089 . Universal Rubber Wahlberg 5122.

Thomsen 5123. Paviors Ltd. 5116.

August, Akt.-Ges. Upjohn Co. 5081.

Uuspärä 5119 .

Vahltelch 5120 .

Töttermann 5062.

Townes 4971. 4972

Dinklage \& Co.

Tehsmer 5118. n Oil Co. of

California 5105 .

United States of

America 5082.

5104. 5107. 5119. Vrbaski 5031.

5093.
Valenta 5030

Valle 5118.

Vasseur 5046

Vaubel 5120.

Vaughan jr., C. W. 5000.

Vaughan, W. R. 4999.

Vesard 4971.

Vereinigte Glaswer

Aachen, Zweig.

niederlassung

Akt.-Ges. der

Spiegelmanufak-

turen und Chem

schen Fabriken
von St. Gobaln,

Chauny \& Clrey 5096.

Verkade 5022.

Vermaas 4991 .

Vialard 5074 .

Virtue 5072.

Vivare!li 4978.

Vivian 5027.

Vodden 5083.

Vogogas Akt.-Ges.
Waksman 5055 .

Walker, J. G. 4976. Wiggins 5004. 5005

Walker, O. J. 5122. 5006 .

Walter 5124. Williamson 4966.

Wallerstein Co.5120. Wiloth 5045 .

Wander, Dr. A. Akt.-Ges. 5081 .

Wang 5065 .

Wanner 5058.

Ward 5057.

Wataghin 4968 .

Waterhouse 5069 .

Waters 5002. 5003.

Watson 5115 .

Watters 5084.

Watteville, de

Webster 5071 .

Wedenejewa 4974 .

Weil, L. 5047

Weil; W. M. 5094 .

Weinbrenner 5116.

Weinstein 5064 .

Weissbecker 5063

Wellcome Foundation Ltd. 5079 .

Wellers 5065 .

Went 4976 .

Wentzel 4966 .

Werf, Van der 5025.

Werner 5060 .

Wilson jr., E. B. 4971.

Wilson jr., R. M. 5101.

Winterfeld 5026 .

Winterstein 5005 .

Wölfel 5118.

Wolf, A. 5106 .

Wolf, J. 5118.

Wolkenstein 4993.

essanen's Konin- Ziffer 5039

kabrieken N. V. 5119 .
Zörner 5085.
Zyka 5076 .

\section{PROF. DR. WALTER TILL}

\section{Die Arzneikunde der Kopten}

Dle Arbelt behandelt in erster Linle dle in den blsher veröffentllchten koptlschen Texten genannten Arznelbestandtelle (d. h. Stoffe, dle zur Bereitung von Hellmitteln verwendet wurden). Diese sind im Hauptteil alphabetisch nach der deutschen Bezeichnung geordnet. Neben den koptlschen Ausdrücken sind grundsätzlich auch die griechlschen und arablschen angeführt, um Vergleiche zu erleichtern. Dle behandelten Stoffe sind, wo möglich, durch die Internatlonal bekannte (latelnische) Bezelchnung gekennzelchnet.

In elnem besonderen Abschnltt sind die In den koptlschen medizinischen Texten genannten Krankheiten erläutert.

Alle in koptlscher Sprache bekannt gewordenen einschlägigen Texte (Rezeptensammlungen), auf die standig verwiesen wird, sind in deutscher Ubersetzung beigegeben, und zwar so, daB jedes gesuchte Rezept lelcht und rasch aufgefunden werden kann. Ausführliche Indizes ermögllchen das rasche Auflinden aller behandelten Gegenstände.

VI und 154 Seiten - Halbleinen DM 49,-

Bestellungen an eine Buchhandlung erbeten

A K A D E M I E - VERLAG GMBH - BERLIN N W 